\title{
Multi-dimensional fragmentomic assay for ultrasensitive early detection of colorectal advanced adenoma and adenocarcinoma
}

\author{
Xiaoji Ma ${ }^{1,2 \dagger}$, Yikuan Chen ${ }^{1,2 \dagger}{ }^{\dagger}$, Wanxiangfu Tang ${ }^{3 \dagger}$, Hua Bao ${ }^{3}$, Shaobo Mo ${ }^{1,2}$, Rui Liu ${ }^{3}$, Shuyu Wu ${ }^{3}$, Hairong Bao ${ }^{3}$, \\ Yaqi Li ${ }^{1,2}$, Long Zhang ${ }^{1,2,4}$, Xue Wu ${ }^{3}$, Sanjun Cai ${ }^{1,2,4}$, Yang Shao ${ }^{3^{*}}$, Fangqi Liu ${ }^{1,2^{*}}$ and Junjie Peng ${ }^{1,2^{*}}$
}

\begin{abstract}
Previous studies on liquid biopsy-based early detection of advanced colorectal adenoma (advCRA) or adenocarcinoma (CRC) were limited by low sensitivity. We performed a prospective study to establish an integrated model using fragmentomic profiles of plasma cell-free DNA (cfDNA) for accurately and cost-effectively detecting early-stage CRC and advCRA. The training cohort enrolled 310 participants, including 149 early-stage CRC patients, 46 advCRA patients and 115 healthy controls. Plasma cfDNA samples were prepared for whole-genome sequencing. An ensemble stacked model differentiating healthy controls from advCRA/early-stage CRC patients was trained using five machine learning models and five cfDNA fragmentomic features based on the training cohort. The model was subsequently validated using an independent test cohort ( $N=311$; including 149 early-stage CRC, 46 advCRA and 116 healthy controls). Our model showed an area under the curve (AUC) of 0.988 for differentiating advCRA/early-stage CRC patients from healthy individuals in an independent test cohort. The model performed even better for identifying early-stage CRC (AUC 0.990) compared to advCRA (AUC 0.982). At 94.8\% specificity, the sensitivities for detecting advCRA and early-stage CRC reached $95.7 \%$ and $98.0 \%$ (0: 94.1\%; I: 98.5\%), respectively. Promisingly, the detection sensitivity has reached $100 \%$ and $97.6 \%$ in early-stage CRC patients with negative fecal occult or CEA blood test results, respectively. Finally, our model maintained promising performances (AUC: $0.982,94.4 \%$ sensitivity at $94.8 \%$ specificity) even when sequencing depth was down-sampled to $1 \mathrm{X}$. Our integrated predictive model demonstrated an unprecedented detection sensitivity for advCRA and early-stage CRC, shedding light on more accurate noninvasive CRC screening in clinical practice.
\end{abstract}

*Correspondence: yang.shao@geneseeq.com; liufq021@163.com; pengjj67@hotmail.com

${ }^{+}$Xiaoji Ma, Yikuan Chen and Wanxiangfu Tang have contributed equally to this work

${ }^{1}$ Department of Colorectal Surgery, Fudan University Shanghai Cancer Center, 270 Dong'an Road, Xuhui, Shanghai 200032, China

${ }^{3}$ Geneseeq Research Institute, Nanjing Geneseeq Technology Inc, Room

1702 Building B Phase I Zhongdan Eco Life Sci Ind Park, Nanjing 210032, Jiangsu, China

Full list of author information is available at the end of the article

\section{To the editor}

Recently, researchers have focused on utilizing plasma cell-free DNA (cfDNA), including cfDNA fragmentomic profiles, to develop noninvasive approaches for detecting solid malignancies such as colorectal adenocarcinoma (CRC) [1-6]. But the limited sensitivities of these current detection methods, by the use of either single molecular feature or single algorithm, reduce their potential utilization in clinical practice, while ensembled stacked machine learning approach can improve robustness and accuracy $[7,8]$. Herein, we constructed a multi-dimensional ensembled stacked machine learning approach, original author(s) and the source, provide a link to the Creative Commons licence, and indicate if changes were made. The images or other third party material in this article are included in the article's Creative Commons licence, unless indicated otherwise in a credit line to the material. If material is not included in the article's Creative Commons licence and your intended use is not permitted by statutory regulation or exceeds the permitted use, you will need to obtain permission directly from the copyright holder. To view a copy of this licence, visit http://creativecommons.org/licenses/by/4.0/. The Creative Commons Public Domain Dedication waiver (http://creativeco mmons.org/publicdomain/zero/1.0/) applies to the data made available in this article, unless otherwise stated in a credit line to the data. 
employing five different base models on five optimized fragmentation features, to provide an ultrasensitive and cost-effective model for detecting early-stage CRC and advanced adenoma (advCRA).

In this study, 149 early-stage colorectal adenocarcinoma (CRC) patients, 46 advCRA patients and 115 healthy volunteers were recruited in the training cohort from a single center, which was used to train the machine learning models (Figs. 1, 2A). To eliminate the potential impact on the predictive power by the different coverages and maximize affordability, WGS data were downsampled to $4 \mathrm{X}$ coverage, unless otherwise noted. The test cohort $(N=311)$, which consisted of 149 early-stage CRC, 46 advCRA patients and 116 healthy controls, was used to evaluate model performances. ROC curves were constructed using five individual features including
Fragment Size Ratio (FSR), Fragment $\underline{\text { Size }}$ Distribution (FSD), EnD Motif (EDM), BreakPoint Motif (BPM) and Copy Number Variation (CNV), as well as the DELFI fragment size pattern [1] and the 4-bp end-motif pattern by Jiang et al. [2], to demonstrate the advantage of using a multi-dimensional ensembled stacked machine learning model approach, as well as adapting existing fragmentation features [7]. Detailed methodology is described in supplementary methods section (Additional file 1).

The ensembled stacked model had a higher AUC (0.988) than base models using any individual feature (AUC range 0.881-0.981), validating the multi-dimensional ensembled stacked approach (Additional file 1: Fig. S1). A similar pattern was observed as the ensembled stacked model had the highest sensitivity for detecting advCRA/early-stage CRC (97.4\%, 95\% CI 94.1-99.2\%)

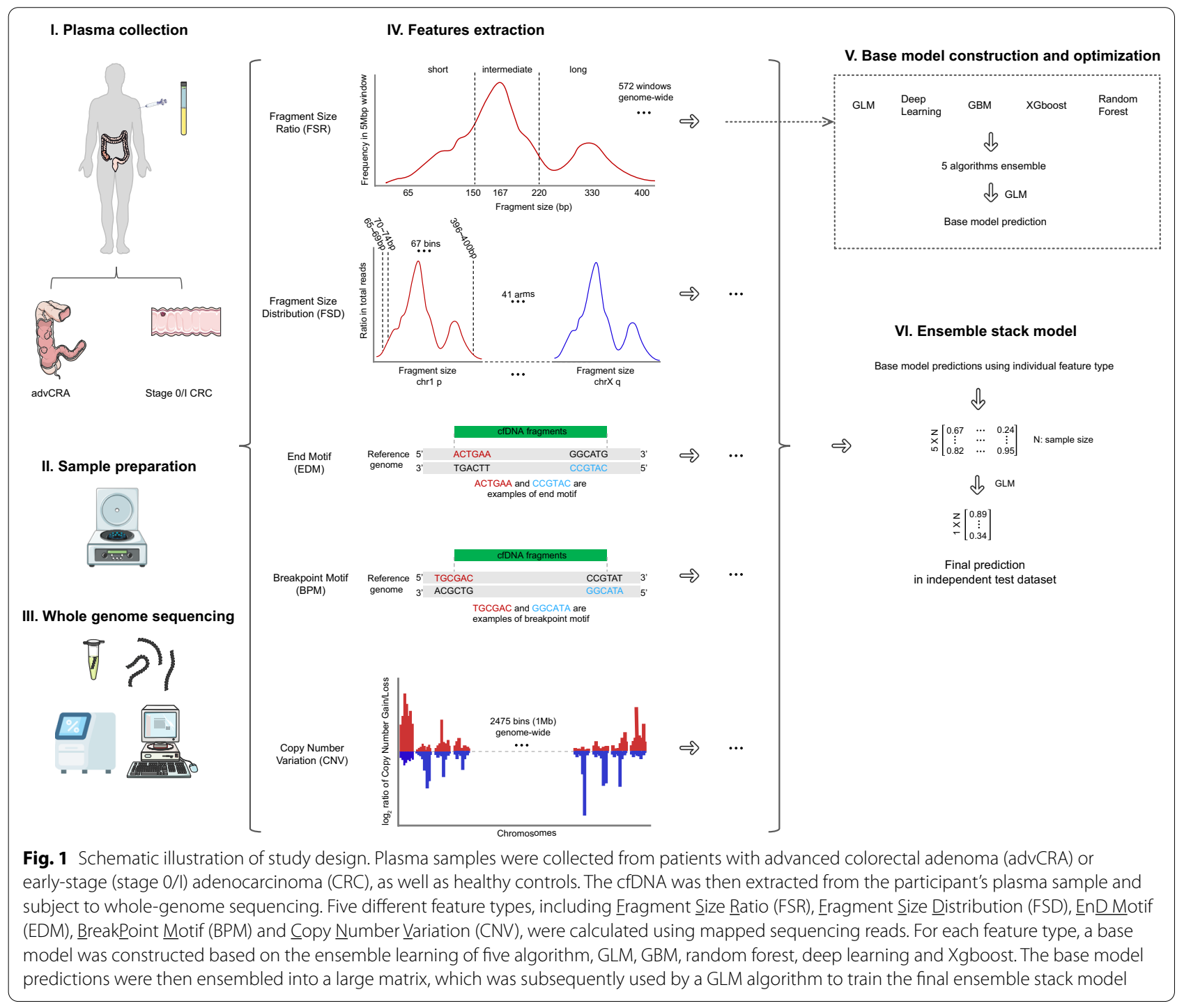




\section{A}

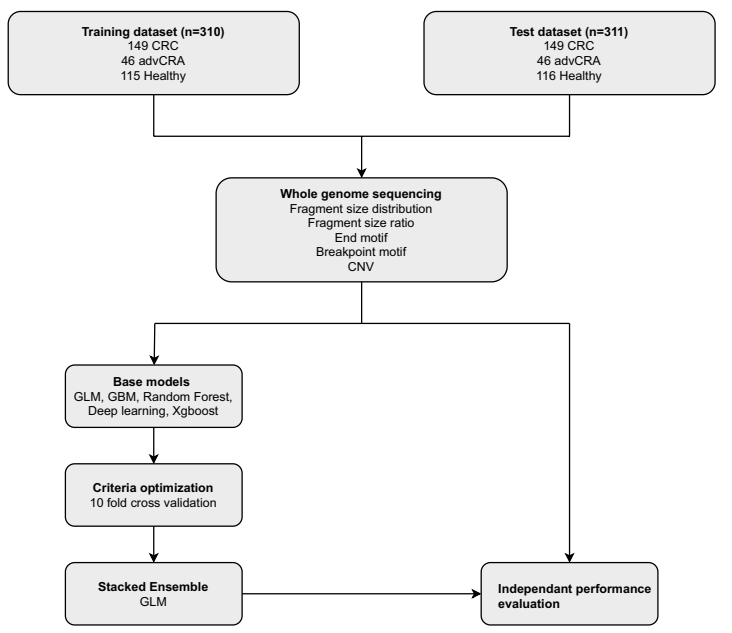

B

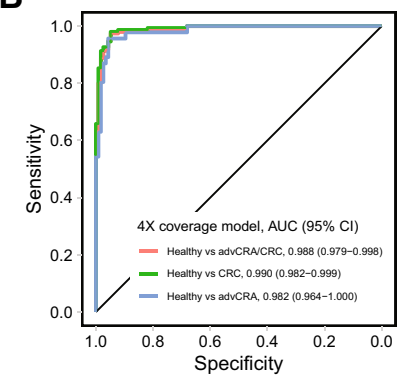

D

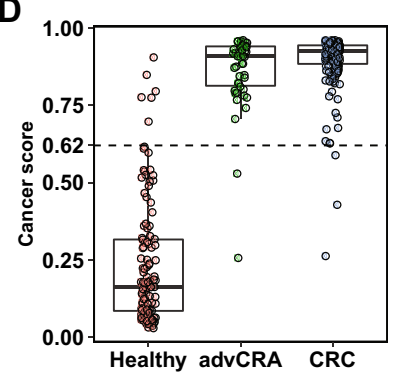

C

\begin{tabular}{|c|c|c|c|}
\hline \multirow{2}{*}{\multicolumn{2}{|c|}{$\begin{array}{c}\text { Healthy vs } \\
\text { advCRA/CRC }\end{array}$}} & \multicolumn{2}{|c|}{ Actual } \\
\hline & & \multirow{2}{*}{$\begin{array}{c}\text { advCRA/CRC } \\
190\end{array}$} & \multirow{2}{*}{$\begin{array}{c}\text { Healthy } \\
6\end{array}$} \\
\hline & advCRA/CRC & & \\
\hline Predict & Healthy & 5 & 110 \\
\hline \multicolumn{2}{|c|}{ Sensitivity $(95 \%$ CI $)$} & \multicolumn{2}{|c|}{$97.4 \%(94.1-99.2 \%)$} \\
\hline \multicolumn{2}{|c|}{ Specificity $(\mathbf{9 5} \% \mathrm{CI})$} & \multicolumn{2}{|c|}{$94.8 \%(89.1-98.1 \%)$} \\
\hline \multirow{2}{*}{\multicolumn{2}{|c|}{ Healthy vs advCRA }} & \multicolumn{2}{|c|}{ Actual } \\
\hline & & advCRA & Healthy \\
\hline \multirow{2}{*}{ Predict } & advCRA & 44 & 6 \\
\hline & Healthy & 2 & 110 \\
\hline \multicolumn{2}{|c|}{ Sensitivity $(95 \% \mathrm{CI})$} & \multicolumn{2}{|c|}{$95.7 \%(85.2-99.5 \%)$} \\
\hline \multicolumn{2}{|c|}{ Specificity $(95 \%$ CI $)$} & \multicolumn{2}{|c|}{$94.8 \%(89.1-98.1 \%)$} \\
\hline \multirow{2}{*}{\multicolumn{2}{|c|}{ Healthy vs CRC }} & \multicolumn{2}{|c|}{ Actual } \\
\hline & & CRC & Healthy \\
\hline \multirow{2}{*}{ Predict } & CRC & 146 & 6 \\
\hline & Healthy & 3 & 110 \\
\hline \multicolumn{2}{|c|}{ Sensitivity $(95 \% \mathrm{CI})$} & \multicolumn{2}{|c|}{$98.0 \%(94.2-99.6 \%)$} \\
\hline \multicolumn{2}{|c|}{ Specificity $(95 \% \mathrm{CI})$} & \multicolumn{2}{|c|}{$94.8 \%$ (89.1-98.1\%) } \\
\hline
\end{tabular}

Fig. 2 Evaluation of ensemble stacked machine learning model. A Graphical representation of datasets composition. The training cohort ( $N=310)$ included 149 early-stage CRC patients, 46 advCRA patients and 115 healthy controls and was used to train the stacked ensemble model. The test cohort $(N=311$ ), which included 149 early-stage CRC patients, 46 advCRA patients and 115 healthy controls, was independently used to evaluate model performances. B ROC curves evaluating the overall performance of the predictive model, which was constructed using 4X coverage WGS data, in distinguishing advCRA/early-stage CRC patients from healthy controls in the test cohort. C Table evaluating model performances in the test dataset. D Boxplots illustrating cancer score distribution in the healthy, advCRA and early-stage CRC groups in the test cohort based on the 4 Xoverage model. The $95 \%$ specificity cutoff for cancer score was 0.62 as shown by the dotted line

compared to all base models (sensitivity range $57.4-$ $89.2 \%)$ at $94.8 \%$ specificity (95\% CI $89.1-98.1 \%)(95 \%$ CI 89.1-98.1\%) (Additional file 1: Fig. S1, Table S1). Additionally, our adaptation to the existing fragmentation features was justified by showing better performances than the original features: the adapted 6-bp EDM feature showed higher AUC (0.981, 95\% CI 0.969-0.993) than the original 4-bp end-motif feature $(0.969,95 \% \mathrm{CI}$ 0.953-0.985), while models using FSR or FSD both had higher AUC (0.881, 95\% CI 0.843-0.919; 0.892, 95\% CI $0.855-0.930)$ than the original DELFI fragment pattern (Additional file 1: Fig. S1).

The stacked model showed better AUC while differentiating early-stage CRC $(0.990,95 \%$ CI 0.981-0.998) than advCRA (0.983, 95\% CI 0.968-0.999) (Fig. 2B). Similarly, the model showed excellent sensitivities for detecting both advCRA (95.7\%, 95\% CI 85.2-99.5\%) and earlystage CRC (98.0\%, 95\% CI 94.2-99.6\%) at the 94.8\% specificity (95\% CI 89.1-98.1\%) (Fig. 2D). The advCRAs more closely resembled early-stage CRCs than healthy controls (Fig. 2C), which was further validated by two additional models (Additional file 1: Fig. S2A, S2B). The current gold standard colonoscopy can be used to histopathologically distinguish advCRA and early-stage CRC following our model's predictions.

We then constructed an ensembled stacked model using the raw depth NGS data (4.7-24.04X, median 9.75X), still showing great performances an identical AUC of 0.988 (95\% CI 0.979-0.997) (Additional file 1: Fig. S3, Table S2). A limit of detection analysis was performed by further down-sampling the $4 \mathrm{X}$ coverage WGS data to $3 \mathrm{X}, 2 \mathrm{X}, 1 \mathrm{X}$ and $0.5 \mathrm{X}$. The down-sampled data was then used to evaluate the $4 \mathrm{X}$ model. The AUCs showed a gradual decrease during the down-sampling process (0.988, 0.987, 0.985, 0.982 and 0.977 for 4X, 3X, 2X, $1 \mathrm{X}$ and $0.5 \mathrm{X}$ data, respectively) (Additional file 1: Fig. S4A).

In summary, our multi-dimensional ensembled stacked model, which uses plasma cfDNA WGS data, showed great potential for accurate noninvasive colorectal cancer screening prior to the current gold standard colonoscopy in clinical practice by demonstrating an unparalleled high sensitivity in detecting early-stage CRC as well as advCRA. However, this study was limited by several factors, namely the relatively small cohort size. The small number of healthy controls within the test cohort can impact the model performance, likely resulting in an 
underestimation of sensitivity. A multicenter, large-scale prospective study is needed to validate the clinical value of our methods further.

\section{Abbreviations}

advCRA: Advanced colorectal adenoma; CRC: Colorectal adenocarcinoma; cfDNA: Cell-free DNA; WGS: Whole-Genome Sequencing; AUC: Area Under the Curve; CNV: Copy number variation; DELFI: DNA EvaLuation of Fragments for early Interception; FSR: Fragment Size Ratio; FSD: Fragment Size Distribution; EDM: EnD Motif; BPM: BreakPoint Motif.

\section{Supplementary Information}

The online version contains supplementary material available at https://doi. org/10.1186/s13045-021-01189-w.

\section{Additional file 1: Supplementary methods. Supplementary Results.} Supplementary Figures. Figure S1. Evaluation of base model using individual features. Figure S2. Evaluation of models distinguishing advCRA from early-stage CRC or healthy controls. Figure S3. Evaluation of model constructed using raw coverage WGS data. Figure S4. Evaluation of a multi-dimensional model detecting advCRA/early-stage CRC. Figure S5. Evaluation of age and gender matched groups in the test cohort. Figure S6. Evaluation of model using 10 -fold cross-validation score of the training cohort. Supplementary Tables. Table S1. Performances evaluation of base models using different features. Table S2. Evaluating performances of model constructed by raw depth data in the test dataset. Table S3. Participant demographics and baseline characteristics. Table S4. Clinical information of the colorectal advanced adenoma (advCRA) and Adenocarcinoma $(C R C)$ patients.

\section{Acknowledgements}

We would like to thank the patients and family members who gave their consent on presenting the data in this study, as well as the investigators and research staff involved in this study.

\section{Authors' contributions}

$J P, F L$ and YS conceptualized and provided guidance throughout the study. $X M$ and $Y C$ performed the experiments, analyzed the data and extensively edited the manuscript. WT performed the computational analysis and wrote the manuscript. XM, YC and WT contributed equally to this work. SM, YL and LZ collected patient samples and documented clinical information. RL, SW and Hairong Bao performed the bioinformatics pipeline. Hua Bao and XW made significant revision to the manuscript. SC provided thoughtful inputs to the study design. All authors read and approved the final manuscript.

\section{Funding}

This work was supported by grants from the National Natural Science Foundation of China (U1932145 to Junjie Peng), Science and Technology Commission of Shanghai Municipality (18401933402 to Junjie Peng), National Natural Science Foundation of China (82002946 to Yaqi Li) and Shanghai Sailing Program (19YF1409500 to Yaqi Li).

\section{Availability of data and materials}

The datasets used and/or analyzed during the current study are available from the corresponding author on reasonable request.

\section{Declarations}

\section{Ethics approval and consent to participate}

All study protocols were approved by the ethics committee of the Fudan University Shanghai Cancer Center, Shanghai Cancer Center Institutional Review
Board (SCCIRB), and in accordance with international standards of good clinical practice. Written informed consents were provided by all patients.

\section{Consent for publication}

The content of this manuscript has not been previously published and is not under consideration for publication elsewhere.

\section{Competing interests}

Wanxiangfu Tang, Hua Bao, Rui Liu, Shuyu Wu, Hairong Bao, Xue Wu and Yang Shao are employees of Nanjing Geneseeq Technology Inc., Nanjing, Jiangsu, China. The remaining authors have nothing to declare.

\section{Author details}

${ }^{1}$ Department of Colorectal Surgery, Fudan University Shanghai Cancer Center, 270 Dong'an Road, Xuhui, Shanghai 200032, China. ${ }^{2}$ Department of Oncology, Shanghai Medical College, Fudan University, Shanghai 200032, China.

${ }^{3}$ Geneseeq Research Institute, Nanjing Geneseeq Technology Inc, Room 1702 Building B Phase I Zhongdan Eco Life Sci Ind Park, Nanjing 210032, Jiangsu, China. ${ }^{4}$ Department of Cancer Institute, Fudan University Shanghai Cancer Center, Fudan University, Shanghai 200032, China.

Received: 10 August 2021 Accepted: 12 October 2021

Published online: 26 October 2021

\section{References}

1. Cristiano S, Leal A, Phallen J, Fiksel J, AdleffV, Bruhm DC, Jensen SO, Medina JE, Hruban C, White JR, et al. Genome-wide cell-free DNA fragmentation in patients with cancer. Nature. 2019;570(7761):385-9.

2. Jiang P, Sun K, Peng W, Cheng SH, Ni M, Yeung PC, Heung MMS, Xie T, Shang $H$, Zhou $Z$, et al. Plasma DNA end-motif profiling as a fragmentomic marker in cancer, pregnancy, and transplantation. Cancer Discov. 2020;10(5):664-73.

3. Rasmussen SL, Krarup HB, Sunesen KG, Johansen MB, Stender MT, Pedersen IS, Madsen PH, Thorlacius-Ussing O. Hypermethylated DNA, a circulating biomarker for colorectal cancer detection. PLOS ONE. 2017;12(7):e0180809.

4. Luo H, Zhao Q, Wei W, Zheng L, Yi S, Li G, Wang W, Sheng H, Pu H, Mo H, et al. Circulating tumor DNA methylation profiles enable early diagnosis, prognosis prediction, and screening for colorectal cancer. Sci Transl Med. 2020;12(524):7533.

5. Jin S, Zhu D, Shao F, Chen S, Guo Y, Li K, Wang Y, Ding R, Gao L, Ma W, et al. Efficient detection and post-surgical monitoring of colon cancer with a multi-marker DNA methylation liquid biopsy. Proc Natl Acad Sci USA. 2021:118(5):985-9.

6. Wan N, Weinberg D, Liu TY, Niehaus K, Ariazi EA, Delubac D, Kannan A, White B, Bailey M, Bertin M, et al. Machine learning enables detection of early-stage colorectal cancer by whole-genome sequencing of plasma cell-free DNA. BMC Cancer. 2019;19(1):832.

7. Zhang C, Ma Y. Ensemble machine learning: methods and applications. New York: Springer; 2012.

8. Kwon H, Park J, Lee Y. Stacking ensemble technique for classifying breast cancer. Healthc Inform Res. 2019;25(4):283-8.

\section{Publisher's Note}

Springer Nature remains neutral with regard to jurisdictional claims in published maps and institutional affiliations. 